\title{
Stability Analysis in a Mutualism System with Linear, Holling Type-II and Functional Response
}

\author{
Solomon $\mathrm{T}^{1}$, Phani Kumar $\mathrm{N}^{2}$, K.V.L.N Acharyulu ${ }^{3}$, Boka Kumsa ${ }^{4}$ \\ and Vishwa Prasad Rao. $S^{5}$ \\ 1,2,4,5 Faculty, Department of Mathematics \\ College of Natural and Computational Sciences, Nekemte, Ethiopia. \\ ${ }^{3}$ Associate Professor, Bapatla Engineering College, Bapatla, A.P, India \\ kvlna@yahoo.com
}

\begin{abstract}
Mutualism is one of the major ecological interactions between two species $\left(N_{l}, N_{2}\right)$, where both species gain benefits via the interactions. In this paper, we have proposed mutualism model for the study of dynamical behaviors of two species $\left(N_{1}, N_{2}\right)$. Mutualisms may exhibit positive feedback in density and qualitative analysis using mathematical modeling. The Lotka-Volterra equations include an expression for population change where the key parameters are a growth term and a maximum population size (carrying capacity). Holling type II has been considered to analyze the proposed model under the local stability of the system numerically by using pplane.
\end{abstract}

Keywords: mutualism, equilibrium point, stability, linearization, phase plane, nullclines analysis and functional response

\section{Introduction}

A population is a group of individuals of the same species that occupy a particular area. Changes in population sizes and composition result from interactions between individuals of the same species, interactions between individuals of different species, interactions with the environment, disease, food supply, etc. Interactions can be predatory, cooperative, mutualistic, commensural, etc. These changes are expressed in terms of birth rates, death rates, immigration rates, and emigration rates. In this paper we concentrate on mutualism. Mutualism is an interaction between different species in which they benefit each other's existence.

The dependency of mutualism refers to how much a species is in need of the interaction, we shall define the notes two different types of dependency that are used by the ecologist Kot, i.e., Facultative Mutualism is a type of mutualism in which the interacting species derive benefit from each other but are not fully dependent that each cannot survive without the other. This is the most common type of mutualism and is exemplified by plants producing fruits which are eaten by birds and the birds helping to dispose the seeds through excretion. Obligate Mutualism is a type of mutualism in which the species involved are in close proximity and interdependent with one another in a way that one cannot survive without the other. A good example of this is between fungus and alga forming lichen [1].

Many researchers date the modern era of population dynamics (sometime called as population ecology) to 1798 and the publication by Malthus of his treatise \An Essay on the Principle of Population" [Malthus, 1798]. Malthus believed that human population grows exponentially while the food supply grows linearly, and ferce competition would naturally ensue. He argued that unless the population is checked by moral restraint or disaster (e.g., disease, famine, or war), widespread poverty and wars would inevitably result. In 1838, Pierre Verhulst proposed his logistic model of population growth, where 
population size is limited by a carrying capacity. Other developers of the modern theory include Gause, Kermack, McKendrick, Leslie, Lotka, May, MacArthur, Ross, and Volterra.

Mathematical models provide a way to design and evaluate protocols to manage and control animal populations, natural resources (e.g., forests), wildlife resources (e.g., fisheries, deer population), and infectious diseases. Management requires predictions and predictions require models. Wright's mathematical theory is based on the premise of a simple two-species mutualism model in which the benefits of mutualism become saturated due to limits posed by handling time. In the models of mutualisms, "type I", "type II" and "type III functional response" refer to the linear and saturating relationships. In this paper, we try to demonstrate the stability of type II functional response.

\section{Assumptions and Model Formulation}

In this paper, the coexistence of two species such as plants $\left(\mathrm{N}_{1}\right)$ and birds $\left(\mathrm{N}_{2}\right)$ are considered. To develop this model the following assumptions have been made.

i) $\mathrm{N}_{1}(\mathrm{t})$ and $\mathrm{N}_{2}(\mathrm{t})$ denotes respectively, the density of plants population and birds population at any instant of time $t$ subject to the non-negative initial condition $\mathrm{N}_{1}(0)=$ $\mathrm{N}_{1}(0) \geq 0$ and $\mathrm{N}_{2}(0)=\mathrm{N}_{2}(0) \geq 0$

ii) The parameter a and $\mathrm{k}_{1}$ be the intrinsic growth rate and environmental carrying capacity of plants population and it is also assumed that the growth of plants population is logistic.

iii) The parameter $\mathrm{c}$ and $\mathrm{k}_{2}$ be the intrinsic growth rate and environmental carrying capacity of birds population and it is also assumed that the growth of birds population is logistic.

iv) The term $\frac{b N_{1}}{1+b h N_{1}}$ is helping rate by birds. This represents the functional response for plants and birds population(Holling type-II functional response) in which the plant's population is benefited and bird's population needs an average of $\mathrm{h}$ time to handle plants item.

v) The parameter' $h$ ' is average time of response (helping time) of birds.

Now by using the above assumptions a mathematical model for coexistences of two species has been developed as follows.

Model-1. The basic model

$$
\begin{aligned}
\frac{d N_{1}}{d t} & =\mathrm{aN}_{1}+\mathrm{bN}_{1} \mathrm{~N}_{2} \\
\frac{d N_{2}}{d t} & =\mathrm{cN}_{1} \mathrm{~N}_{2}+\mathrm{dN}_{2}
\end{aligned}
$$

Model-2.(Incorporating carrying capacity)

$$
\begin{aligned}
& \quad \frac{d N_{1}}{d t}=\mathrm{aN}_{1}\left(1-\frac{N_{1}}{K_{1}}\right)+\mathrm{bN}_{1} \mathrm{~N}_{2} \\
& \frac{d N_{1}}{d t}=\mathrm{cN}_{1} \mathrm{~N}_{2}+\mathrm{dN}_{2}\left(1-\frac{N_{2}}{K_{2}}\right)
\end{aligned}
$$

Model-3(Holling type II-functional response)

$$
\frac{d N_{1}}{d t}=\mathrm{a} \mathrm{N}_{1}\left(1-\frac{N_{1}}{K_{1}}\right)+\left(\frac{b N_{1}}{1+b h N_{1}}\right) \mathrm{N}_{2}
$$




$$
\frac{d N_{2}}{d t}=\mathrm{dN}_{2}\left(1-\frac{N_{2}}{K_{2}}\right)+\mathrm{cN}_{1} \mathrm{~N}_{2}
$$

\section{Equilibrium Points}

For establishing the stability of the above models, the equilibrium points are very important to analyze any model. Now the possible equilibrium points of the above models are given as follows,

In this model-1 we have only two equilibrium points (i). The first equilibrium point is the trivial equilibrium point i.e., $\mathrm{E}_{0}=(0,0)$ (ii). The second equilibrium point is negative equilibrium point which is biologically not interested.

In this paper we focused only on model- 2 and model-3 for analyzing the system. The equilibrium points of system (2) are (i). The trivial equilibrium point at which no population exists i.e., $\mathrm{E}_{10}=(0,0)$ (ii). The equilibrium point $\mathrm{E}_{11}=(1,0)$ in which only population one exists (iii).The equilibrium point $\mathrm{E}_{12}=(0,1)$, in which only population two exists and (iv).The equilibrium point $\mathrm{E}_{13}=\left(\frac{a d+d b k_{2}}{a d-b c k_{1} k_{2}}, \frac{a d+a c k_{1}}{a d-b c k_{1} k_{2}}\right)$ for ad $>b c k_{1} \mathrm{k}_{2}$ which shows the coexistence of population.

Before going to determine the critical points of the system (3), let us nondimensionalise the system by defining

$\alpha=\frac{b k_{2}}{\mathrm{a}}, \beta=\mathrm{bh} \mathrm{k}, \gamma=\frac{d}{\mathrm{a}}$ and $\delta=\frac{k_{1} c}{\mathrm{a}}$

Then system (2) becomes

$$
\begin{aligned}
& \frac{d N_{1}}{d t}=\mathrm{N}_{1}\left(1-\mathrm{N}_{1}\right)+\frac{\alpha N_{1} N_{2}}{1+\beta N_{1}} \\
& \frac{d N_{2}}{d t}=\gamma \mathrm{N}_{2}\left(1-\mathrm{N}_{2}\right)+\delta \mathrm{N}_{1} \mathrm{~N}_{2}
\end{aligned}
$$

The trivial equilibrium point $\mathrm{E}_{20}=(0,0)$, in which there is no population. The equilibrium point $\mathrm{E}_{21}=(0,1)$, only second population exists. The equilibrium points $\mathrm{E}_{22}=(1,0)$,only first population exists. The equilibrium point $\mathrm{E}_{23}=\left(\frac{A+\sqrt{B}}{2 \alpha \beta}, 1+\frac{\alpha}{\gamma}\left(\frac{A+\sqrt{B}}{2 \alpha \beta}\right)\right)$ occurs only when $\alpha \beta>0$, Coexistence of both populations exist in the case of $\mathrm{A}=\gamma \beta-$ $\gamma+\alpha \delta$ and $\mathrm{B}=(\gamma \beta-\gamma+\alpha \delta)^{2}+4(\gamma+\gamma \alpha)(\alpha \beta)$.

\section{Stability Analysis}

In this section, let us consider the stability analysis of the deterministic system (2). The stability analysis can be determined by the nature of the eigen values of the community matrix around each equilibrium $m$ points.

The community matrix of the system (1) around the equilibrium point is

$$
\mathrm{J}=\left(\begin{array}{cc}
1-2 \mathrm{~N}_{1}+\frac{b k_{2} \mathrm{~N}_{2}}{\mathrm{a}} & \frac{b k_{2} N_{1}}{a} \\
\frac{c k_{1} N_{2}}{a} & \frac{c k_{1} N_{1}}{a}+\frac{d}{a}-2 \frac{d N_{2}}{a}
\end{array}\right)
$$

Theorem.1. The trivial equilibrium point $E_{10}$ and the boundary equilibrium points $E_{11}$ and $E_{12}$ are unstable.

Proof. The community matrix at $\mathrm{E}_{10}$ is, $\mathrm{JE}_{10}=\left(\begin{array}{ll}1 & 0 \\ 0 & \frac{d}{a}\end{array}\right)$

It has an eigen values $\lambda_{1}=1$ and $\lambda_{2}=\frac{d}{a}$. This indicates that the trivial equilibrium point is unstable. The community matrix at $\mathrm{E}_{11}$ is, 
$\mathrm{J} \mathrm{E}_{11}=\left(\begin{array}{cc}-1 & \frac{b k_{2}}{a} \\ 0 & \frac{c k_{1}+d}{a}\end{array}\right)$ and it has an eigen values $\lambda_{1}=-1$ and $\lambda_{2}=\frac{c k_{1}+d}{a}$ which shows that $\mathrm{E}_{11}$ is saddle point. It is stable in the direction of $\mathrm{N}_{1}$ and unstable in the direction of $\mathrm{N}_{2}$. Now for the third equilibrium point $\mathrm{E}_{12}$ the corresponding community matrix of $\mathrm{J}_{\mathrm{E} 12}$ $=\left(\begin{array}{cc}1+\frac{b k_{2}}{a} & 0 \\ \frac{c k_{1}}{a} & \frac{-d}{a}\end{array}\right)$. The corresponding eigen values are $\lambda_{1}=1+\frac{b k_{2}}{a} \quad$ and $\lambda_{2}=\frac{-d}{a}$ which shows that $E_{12}$ is saddle point. It has stable in the direction of $\mathrm{N}_{2}$ and unstable in the direction of $\mathrm{N}_{1}$.

Theorem -2 The equilibrium point of $\mathrm{E}_{13}$ is stable only if determinant of its community matrix is positive, otherwise unstable.

Proof. The community matrix of the $\mathrm{E}_{13}$ is given as $\mathbf{J} \boldsymbol{E}_{\mathbf{1 3}}=\left(\begin{array}{cc}u & v \\ w & s\end{array}\right)$, where u=-ad-dbk$k_{2}<$ 0

$\mathrm{S}=-\mathrm{cadk}_{1}-\mathrm{ad}^{2}<0, \mathrm{w}=\frac{c d k_{1}+c^{2} k_{1}^{2}}{a d-b c k_{1} k_{2}}>0$ and $\mathrm{v}=\frac{a d b k_{2}+d b^{2} k_{2}^{2}}{a\left(a d-b c k_{1} k_{2}\right)}>0$. Now Trace $(\mathrm{T})=\mathrm{u}+\mathrm{s}$ $<0$ and determinant $(\mathrm{D})=\mathrm{us}-\mathrm{vw}$

Then we have different possibilities for $\mathrm{D}$ to find the eigen values.

(i).For $\mathrm{D}>0$, from the characteristics equation we have $\lambda=\frac{T \pm \sqrt{T^{2}-4 D}}{2}$. Again we have three sub cases,

If $\mathrm{T}^{2}-4 \mathrm{D}>0$, both Eigen values are real and negative which imply that it is stable.

If $\mathrm{T}^{2}-4 \mathrm{D}<0$, we can get complex Eigen values in which the real part is negative. The stability in this case is sink which is stable. And if $T^{2}-4 D=0$, we can get repeated root which has negative values. In this case, all Eigen values are negative which imply that at this point, the equilibrium points are stable.

(ii).For $\mathrm{D}<0$, from the characteristics equation we can get that $\lambda_{1}>0$ and $\lambda_{2}<0$ which is saddle point. That is unstable from the $\mathrm{N}_{1}$ direction and stable from $\mathrm{N}_{2}$ direction.

\section{Numerical Illustration}

In this section, we will see the numerical illustrations of model-2 and model-3 and also their dynamical behaviors using MatLab called pplane. Due to unavailability of real data of all parameters associated with the model, the hypothetical values to the different parameters have been considered as follows:

Model-2: (Incorporating carrying capacity)

Consider the parameter values $\mathrm{a}=1, \mathrm{~b}=1, \mathrm{c}=0.5, \mathrm{~d}=2, \mathrm{k}_{1}=1$ and $\mathrm{k}_{2}=1$ From this, we can see that, for the trivial equilibrium point $\mathrm{E}_{10}$, we have $\lambda_{1}=1>0$ and $\lambda_{2}=2>0$. So according to theorem (1) the trivial equilibrium is unstable. And also from the same data, for the boundary equilibrium points $E_{11}$, we have $\lambda_{1}=-1<0$ and $\lambda_{2}=2.5>0$ and for $E_{12}$ ,again we have $\lambda_{1}=1.5>0$ and $\lambda_{2}=-2<0$ which imply that both are saddle points according to theorem (1), (a).At $E_{11}$,only $\mathrm{N}_{1}$ exists which is stable and $\mathrm{N}_{2}$ is washed out (b). At $\mathrm{E}_{12}$, only $\mathrm{N}_{2}$ exists in stable way and $\mathrm{N}_{1}$ extincts (see Figures 1.1-1.3)

Again for the parameter values, $\mathrm{a}=0.5, \mathrm{~b}=0.8, \mathrm{c}=0.5, \mathrm{~d}=2, \mathrm{k}_{1}=0.5, \mathrm{k}_{2}=1$ Then, for the equilibrium point $\mathrm{E}_{3}=(1.6,1.4)$, we have $\lambda_{1}=-1<0$ and $\lambda_{2}=-3<0$ so according to theorem(2) the equilibrium point $E_{3}$ is stable and Figure 1.4 show that, at this point both plants and birds population exist and they can survive by helping each other. There is no extinction of population. 
Model -3 (Holling type II-functional response)

Consider the parameter values $\mathrm{a}=3, \mathrm{~b}=2, \mathrm{c}=1, \mathrm{~d}=3, \mathrm{k}_{1}=2, \mathrm{k}_{2}=1$ and $\mathrm{h}=1$ Then from this data, let's show the numerical illustration at the trivial and boundary equilibrium points $\left(\mathrm{E}_{20}, \mathrm{E}_{21}\right.$ and $\left.\mathrm{E}_{22}\right)$. By using this data at $\mathrm{E}_{20}$, we have $\lambda_{1}=1>0$ and $\lambda_{2}=0.1>0$ which is unstable. Originally at $\mathrm{E}_{20}$, we have no population and after we perturb small population, they start to grow. And also with same parameters. When we check the behavior of plants and birds population at the boundary equilibrium point at $E_{21}, \lambda_{1}=$ $2>0$ and $\lambda_{2}=-1<0$ and at $\mathrm{E}_{22}$, we have $\lambda_{1}=-1<0$ and $\lambda_{2}=0.3>0$. Therefore the boundary equilibrium point $E_{21}$ and $E_{22}$ are saddle points which are unstable. From $E_{21}$ we can see that we have only population two which is stable but after we add small amount of population one, they start to grow and become saturated. From $\mathrm{E}_{22}$ we can understand that, we have only the first population which is stable but after we add small amount of the second population, they start to grow and after a time t they become saturated. See Figure 2.1-2.3

Again for the set of parametric values of $\mathrm{a}=2, \mathrm{~b}=1, \mathrm{c}=0.5, \mathrm{~d}=2, \mathrm{k}_{1}=1, \mathrm{k}_{2}=2$, and $\mathrm{h}=1 \mathrm{It}$ is seen that, $\mathrm{E}_{23}=(2.27,1.6)$, then we have $\lambda_{1}=-2.66<0$ and $\lambda_{2}=-10.733<0$. So fourth equilibrium point is stable. In this case there are different cases for stability depending on trace and determinant, but we are checking only for the eigen values real and distinct. In this case both plants and birds population exist together Figure 2.4.

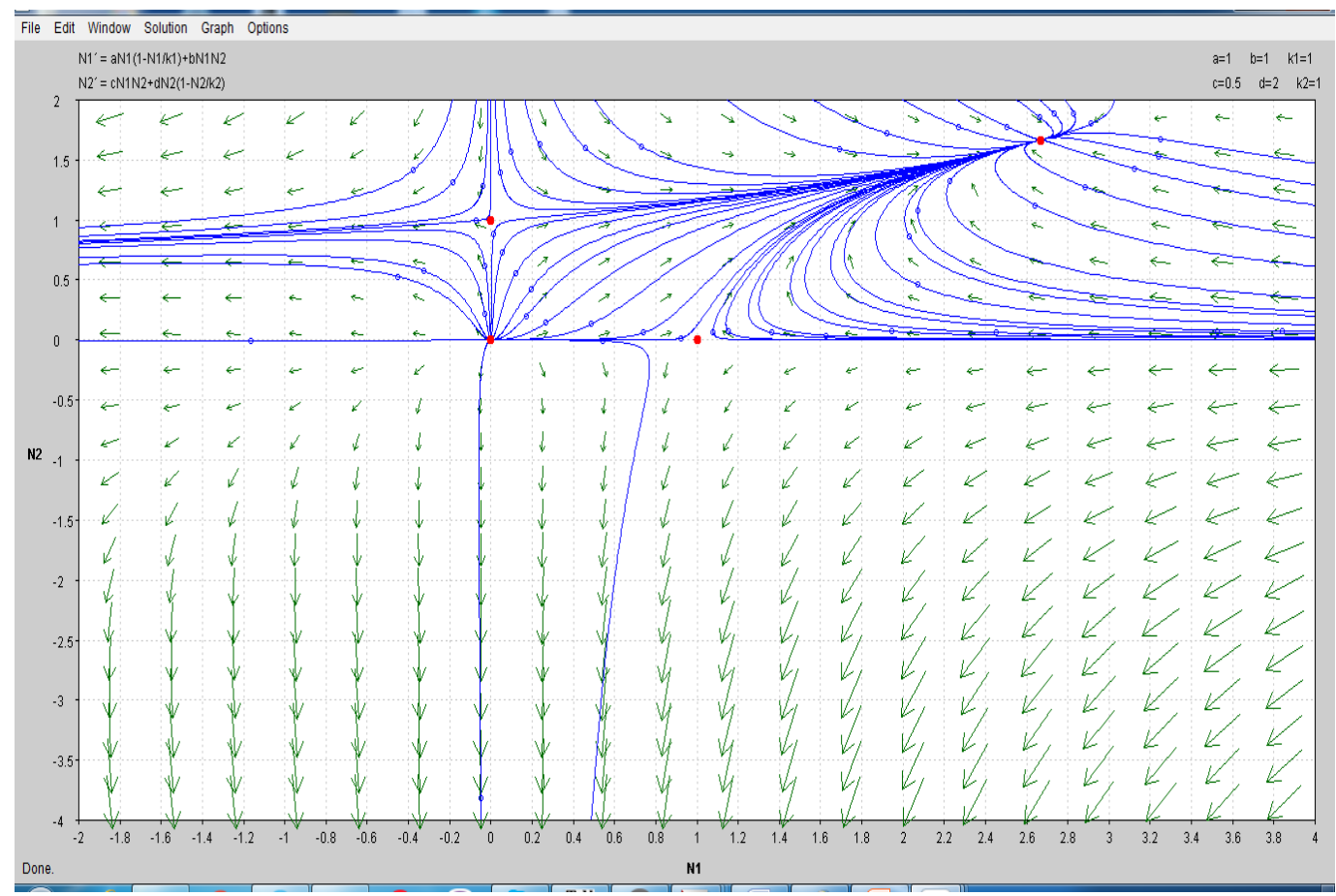

Figure 1. The Phase Plane Analysis of Model -2 with the Parameters $a=1, b=1, c=0.5, d=2, k_{1}=1$ and $k_{2}=1$ 


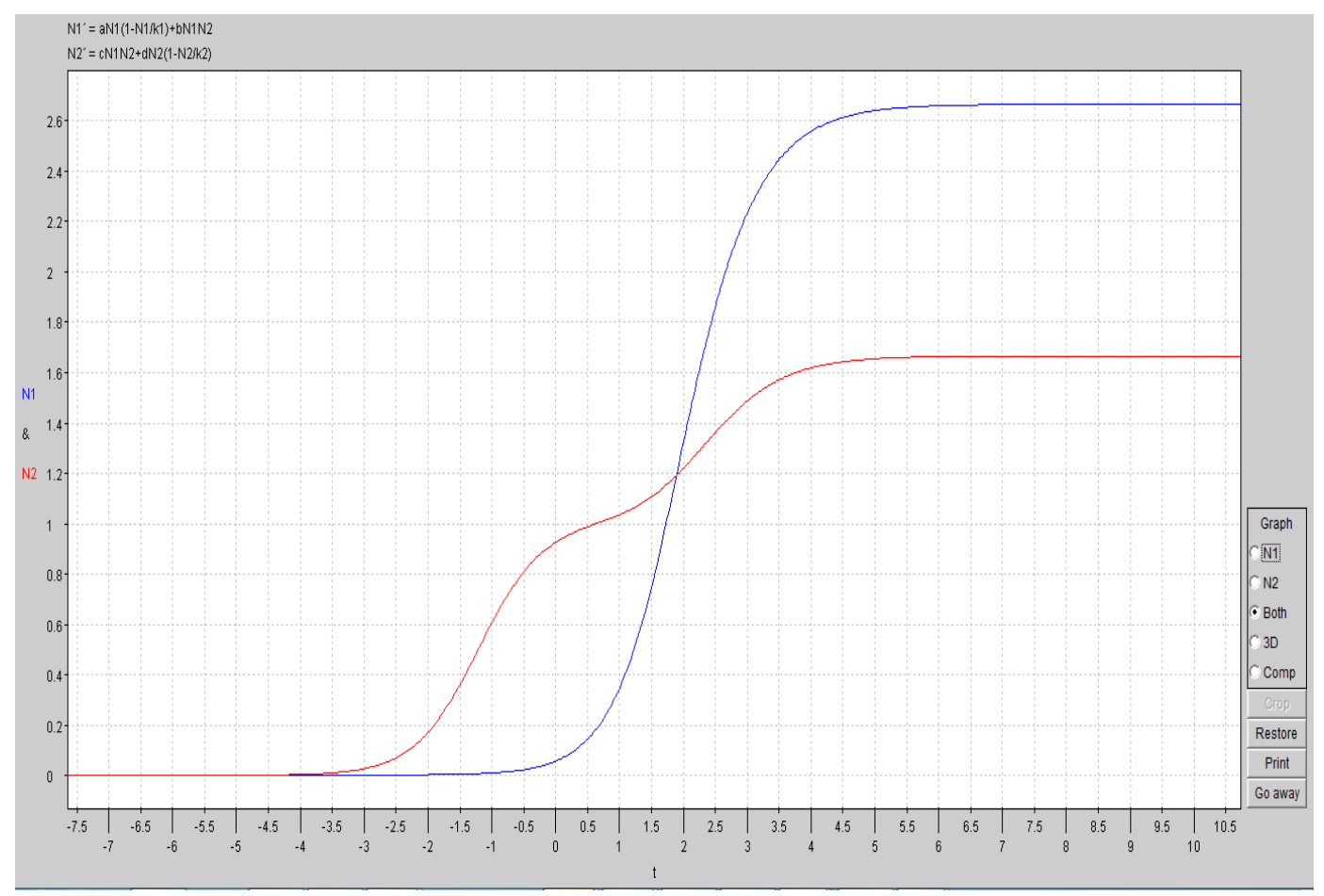

Figure 1.1. The Stability Analysis of $N_{1}$ and $N_{2}$ versus Time $t$ at the Trivial Equilibrium Point $E_{10}$ with the Parameters $a=1, b=1, c=0.5, d=2, k_{1}=1$ and $k_{2}=1$

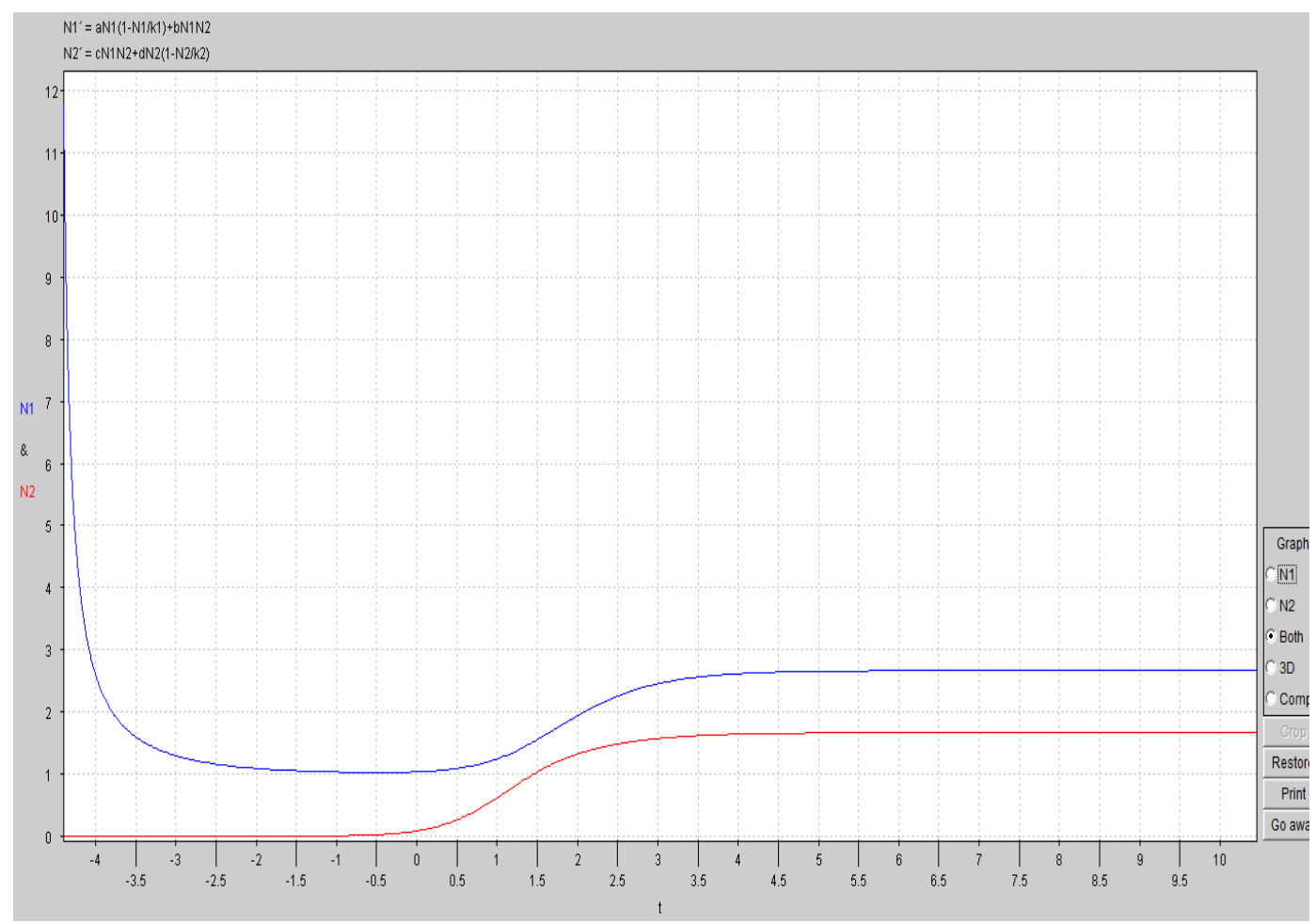

Figure 1.2. The Stability Analysis of $N_{1}$ and $N_{2}$ versus Time $t$ at the Equilibrium Point $E_{11}$ with the Parameters $a=1, b=1, c=0.5, d=2, k_{1}=1$ and $k_{2}=1$ 


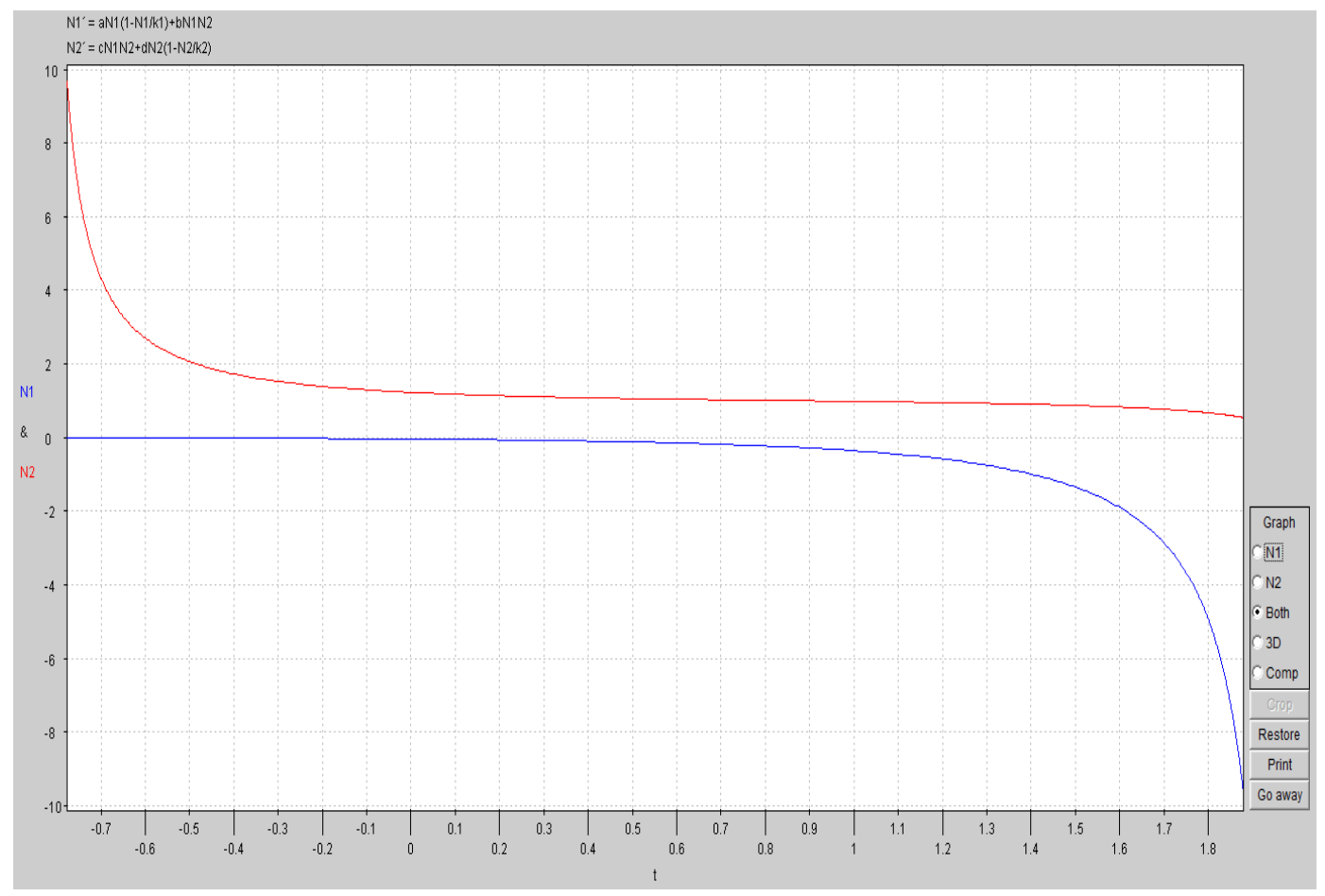

Figure 1.3. The Stability Analysis of $N_{1}$ and $N_{2}$ versus Time $t$ at the Equilibrium Point $E_{12}$ with the Parameters $a=1, b=1, c=0.5, d=2, k_{1}=1$ and $k_{2}=1$

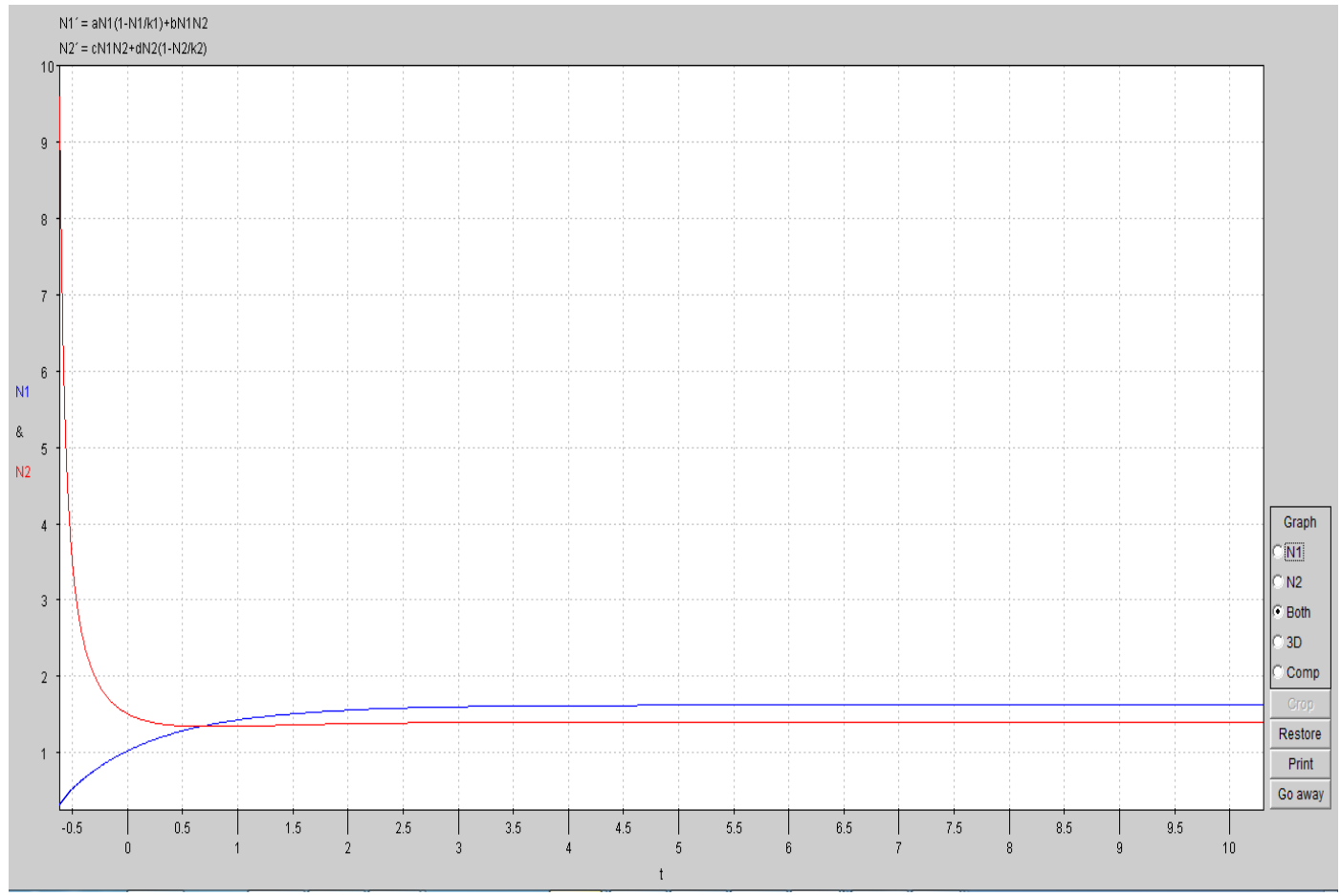

Figure 1.4. The Stability Analysis of $\mathrm{N}_{1}$ and $\mathrm{N}_{2}$ versus Time at the Equilibrium Point $\mathrm{E}_{13}$ at the Parameters, $\mathrm{a}=0.5, \mathrm{~b}=0.8, \mathrm{c}=0.5, \mathrm{~d}=2, \mathrm{k}_{1}=0.5$, $k_{2}=1$ 


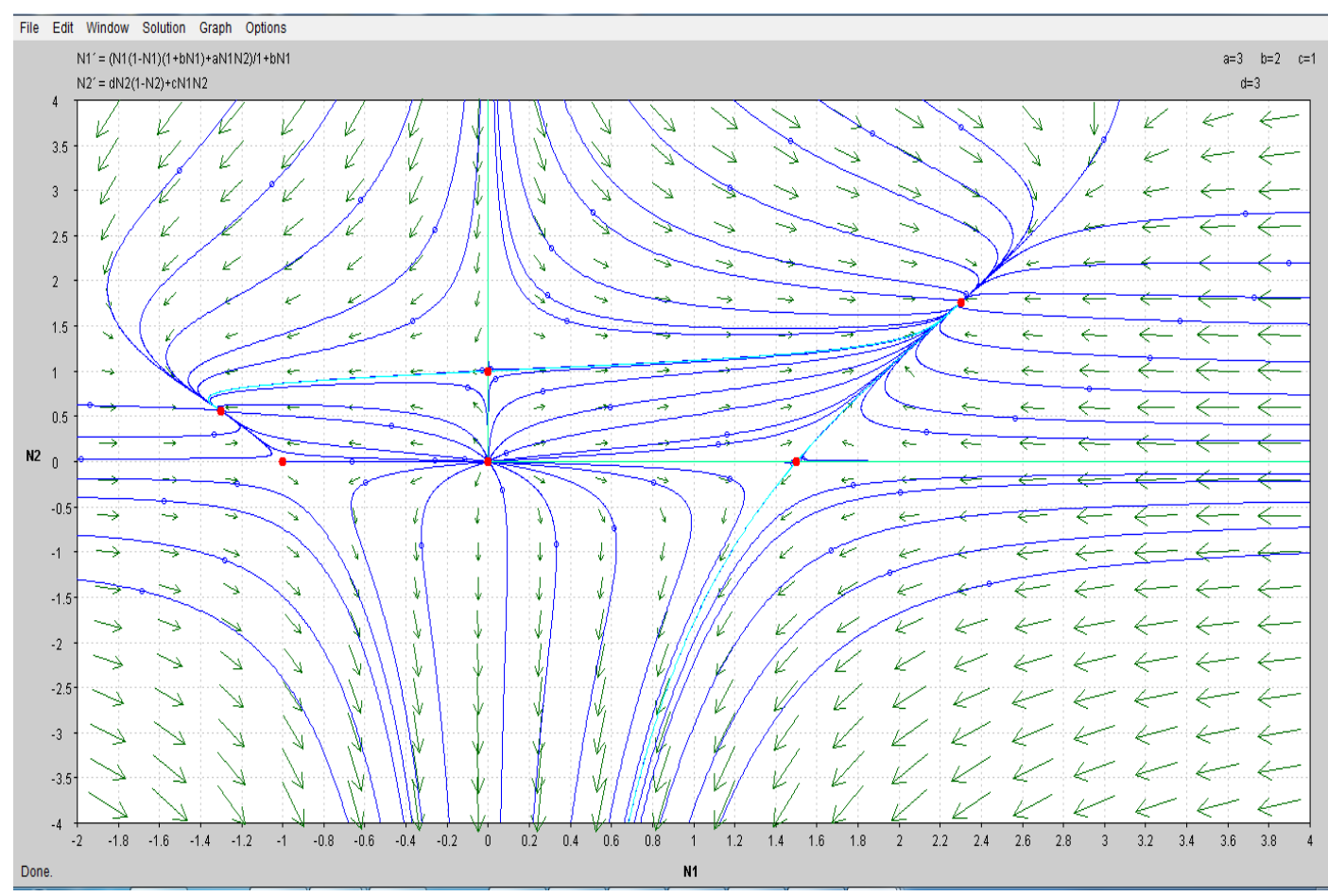

Figure 2. The Phase Plane Analysis of Model -3 with the Parameters $a=3$, $b=2, c=1, d=3, k_{1}=2, k_{2}=1$ and $h=1$

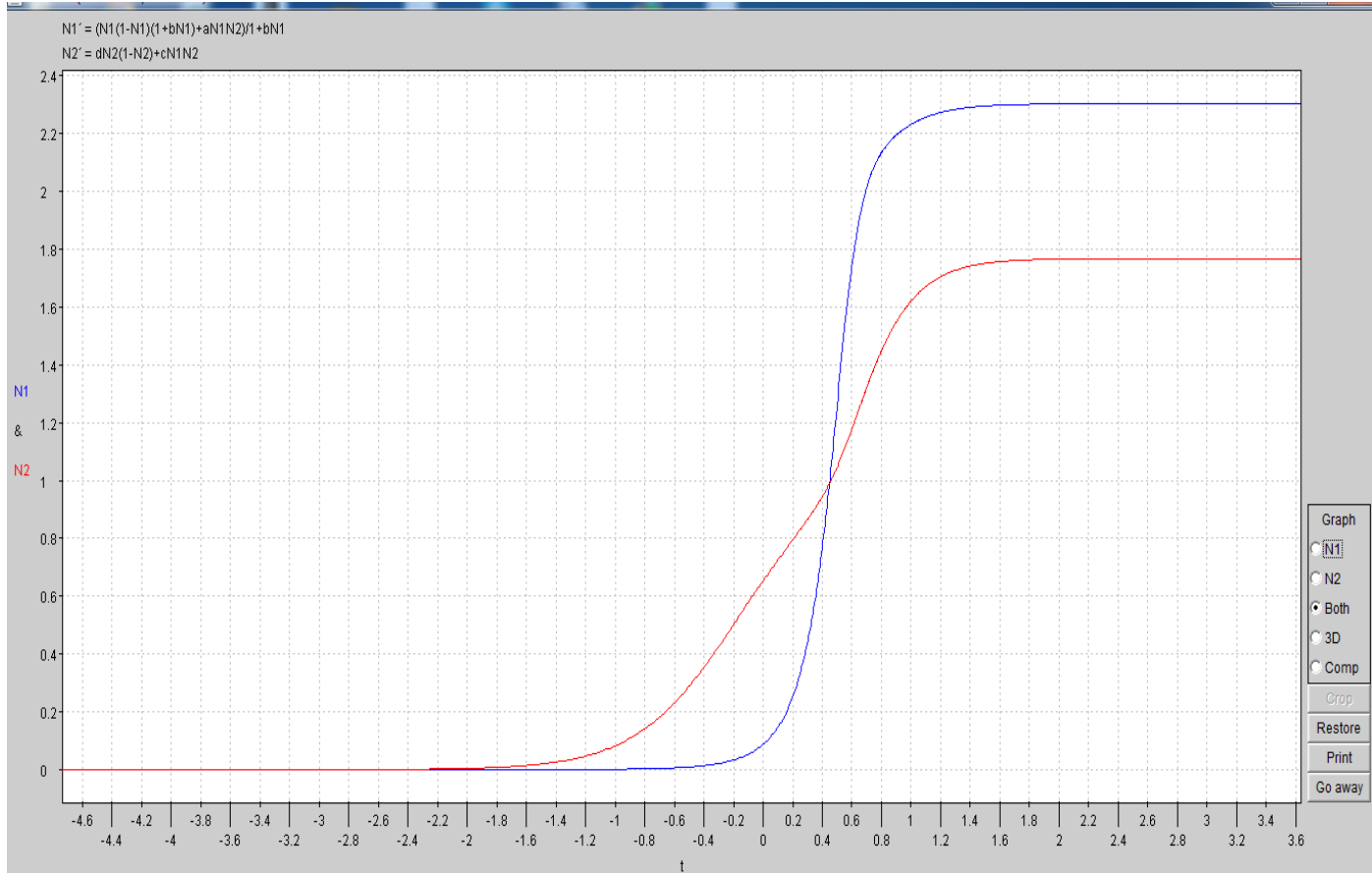

Figure 2.1. The Stability Analysis of $N_{1}$ and $N_{2}$ versus Time $t$ at the Trivial Equilibrium Point $E_{20}$ with the Parameters $a=3, b=2, c=1, d=3, k_{1}=2, k_{2}=1$ and $h=1$ 


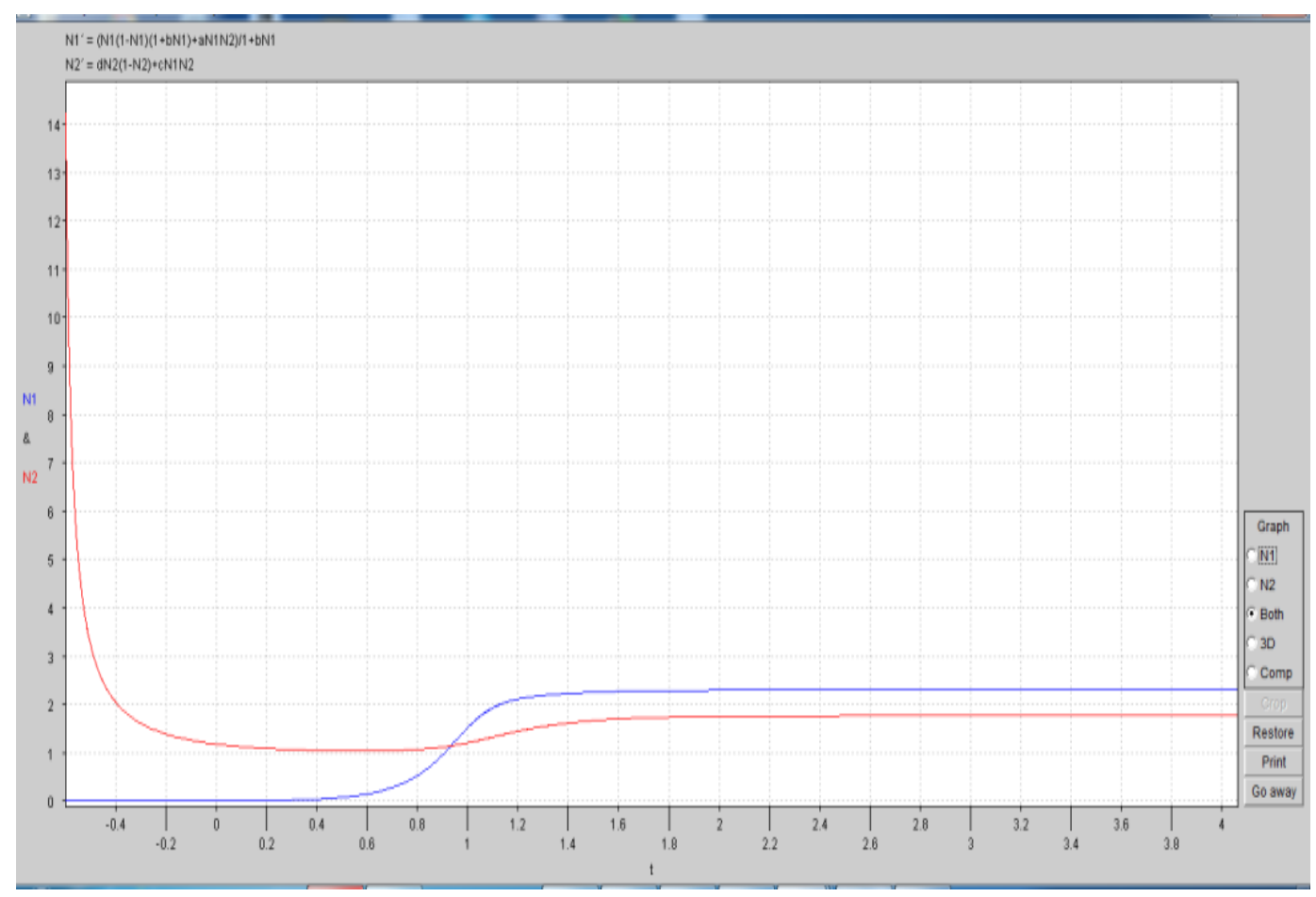

Figure 2.2. The Stability Analysis of $\mathrm{N}_{1}$ and $\mathrm{N}_{2}$ versus Time $t$ at the Equilibrium Point $E_{21}$ with the Parameters $a=3, b=2, c=1, d=3, k_{1}=2, k_{2}=1$ and $h=1$

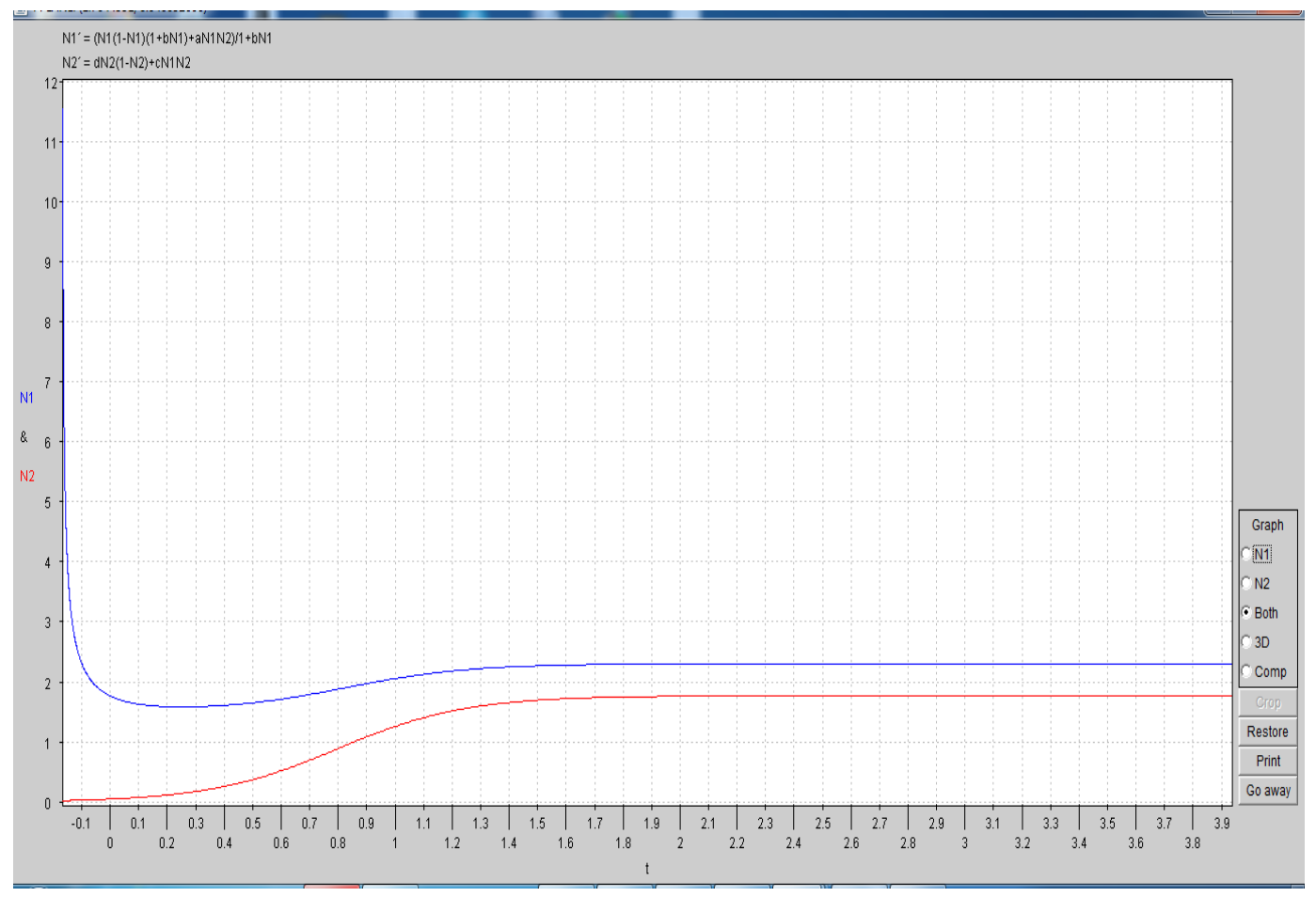

Figure 2.3. The Stability Analysis of $\mathrm{N}_{1}$ and $\mathrm{N}_{2}$ versus Time $t$ at the

Equilibrium point $E_{22}$ with the Parameters $a=3, b=2, c=1, d=3, k_{1}=2, k_{2}=1$ and $h=1$ 


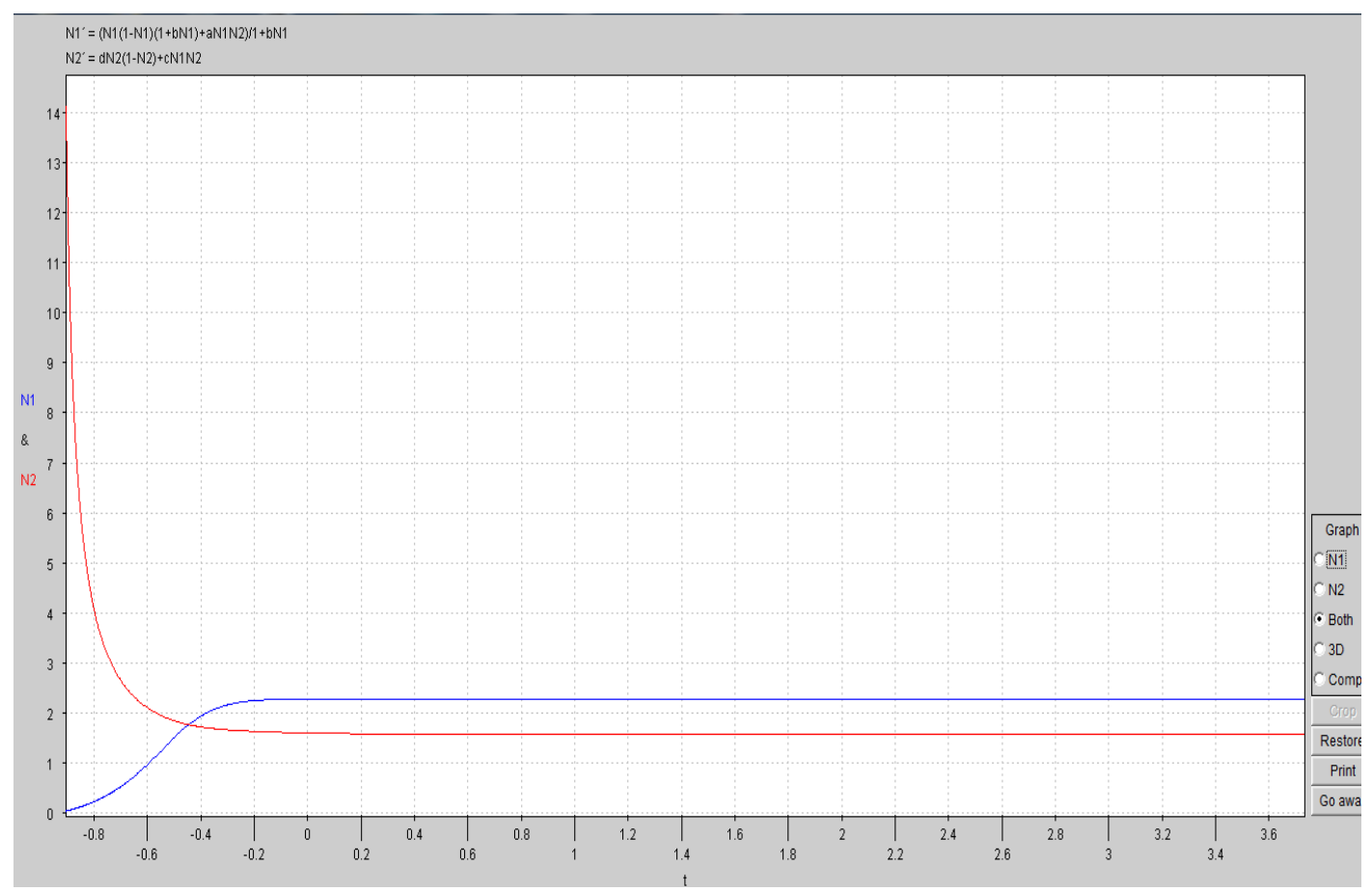

Figure 2.4. The Stability Analysis of $N_{1}$ and $N_{2}$ versus Time $t$ at the Equilibrium Point $E_{23}$ with the Parameters $a=3, b=2, c=1, d=3, k_{1}=2, k_{2}=1$ and $h=1$

\section{Nullclines Analysis}

The analysis of nullclines gives the more clear visualization of phase plane analysis of the models. By dividing the phase plane into regions, then only we can analyse the behavior of our dynamics.

Figure 3. Shows the nullclines analysis of model-2

Figure 4. Shows the nullclines analysis of model-3

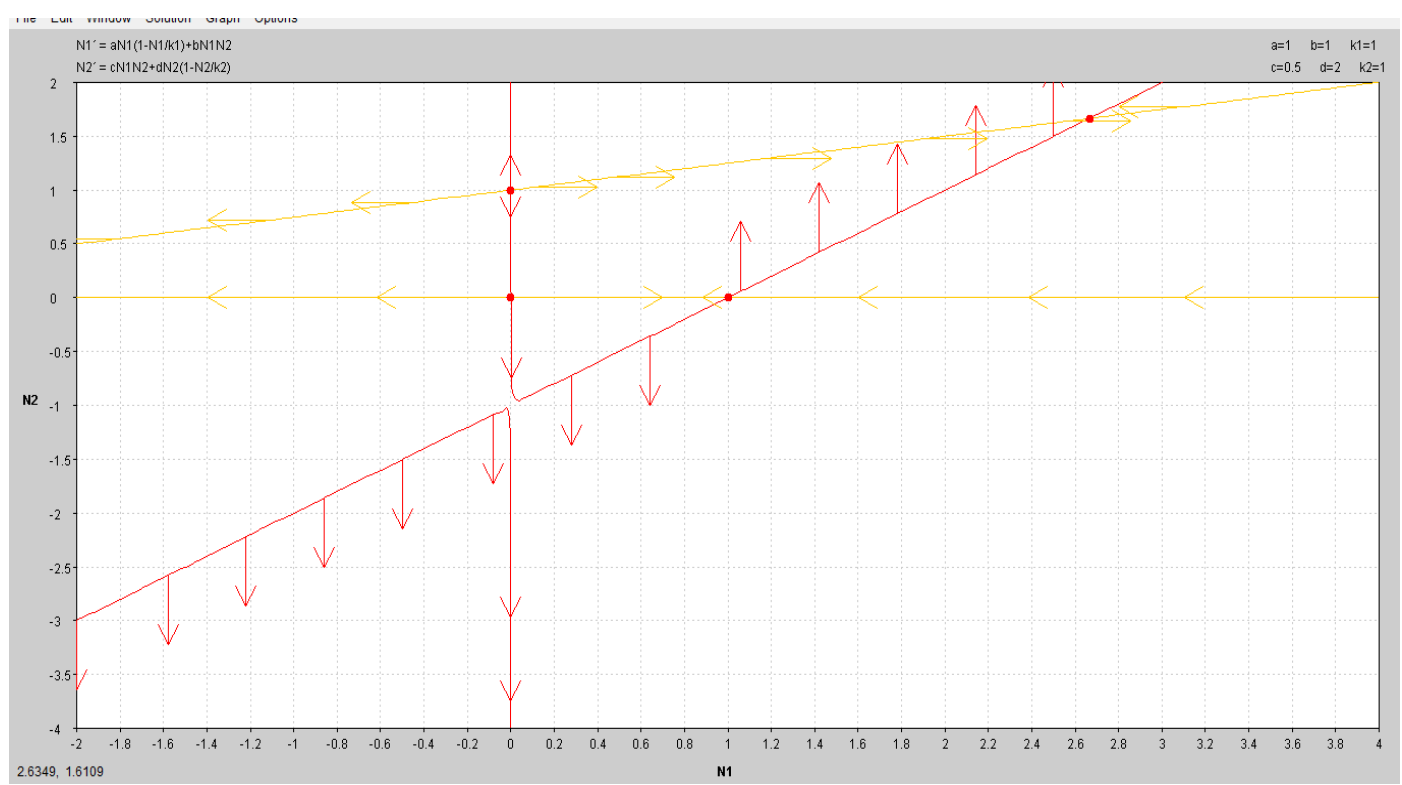

Figure 3. The Nullclines of Model -1(Incorporating Carrying Capacity) with the Parameters $a=1, b=1, c=0.5, d=2, k_{1}=1$ and $k_{2}=1$ 


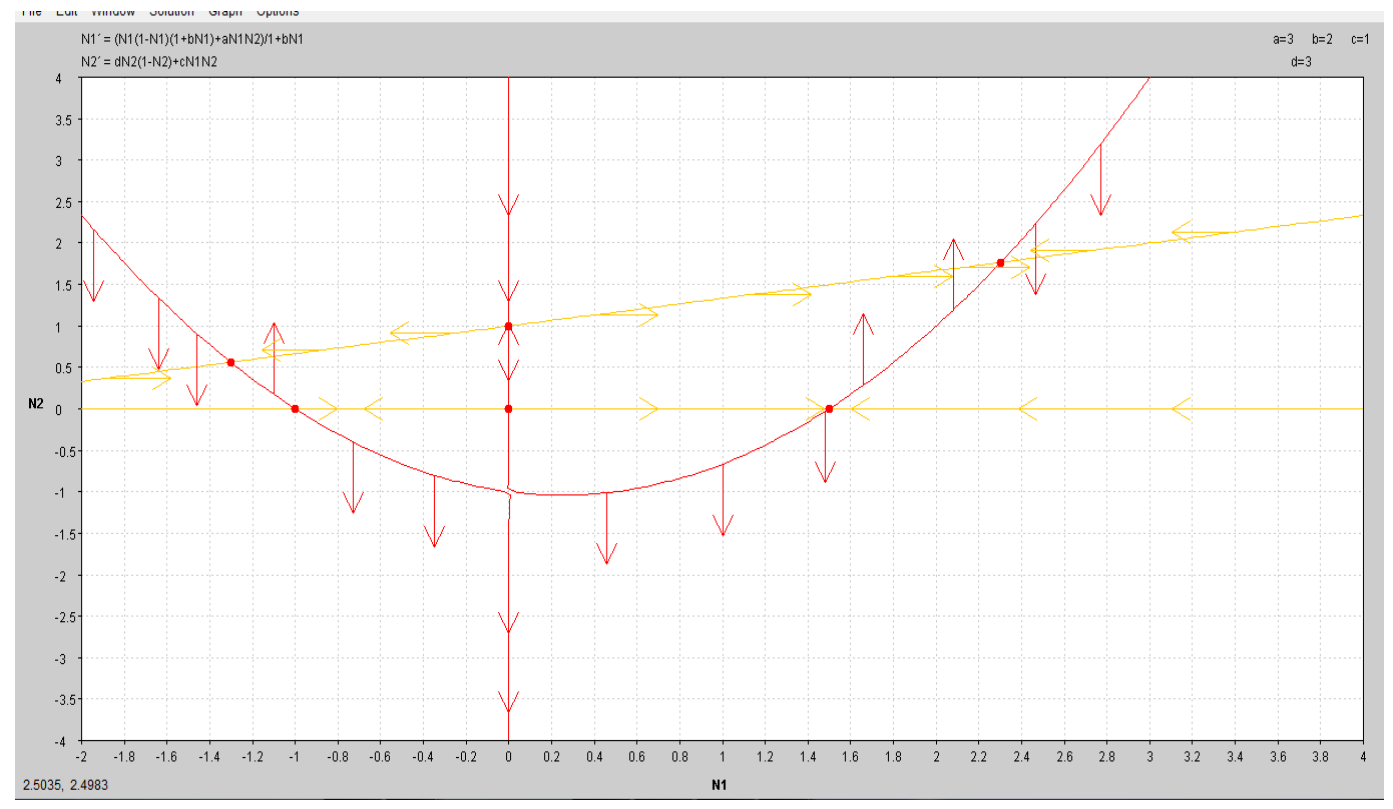

Figure 4. The Nullclines Analysis of Model-2 (Holling type II functional response) 3 with the Parameters $a=3, b=2, c=1, d=3, k_{1}=2, k_{2}=1$ and $h=1$

\section{Conclusions}

In this paper, we have proposed a mathematical model of mutualism in which the coexistence of two species (plants and birds) is investigated. Few different models are considered and the stability behavior of two species of mutualism discussed around each equilibrium points. We illustrated the models numerically by using different parameters. We also saw the nullcline analysis of each model of plant's population versus bird's population. The trajectories of the model at each equilibrium point are illustrated with respect to (i). Plant's population versus time \& (ii). Bird's population versus time for each model.

\section{References}

[1] J. D. Skellam, "Random dispersal in theoretical population", Miometrika, vol. 38, (1951).

[2] H. L. Smith, "The interaction of steady state and Hopf bifurcations in a two-predator-one- prey competition model", SIAM Journal on Applied Mathematics, vol. 42, (1982).

[3] H. L. Smith and P. Waltman, "The theory of the chemostat", Cambridge University Press Cambridge, UK, (1995).

[4] J. M. Smith, "Mathematical ideas in biology", Cambridge University Press, (1968).

[5] J. Smoller, "Shock waves and reaction-diusion equations", Springer, (1994).

[6] M. E. Solomon, "The natural control of animal populations", The Journal of Animal Ecology, vol. 18, (1949).

[7] L. Sompayrac, "How the immune system works", Blackwell Publishing, (2003).

[8] N. Phanikumar, "Some mathematical models of ecological commensalim", Ph.D. Thesis, ANU, (2010).

[9] A. J. Lotka, "Elements of Physical Biology", Williams and Wilking, Baltimore, (1925).

[10] W. J. Meyer, "Concepts of Mathematical Modeling”, Mc. Graw-Hill, (1985). 
International Journal of Advanced Science and Technology Vol.103 (2017) 